\title{
CASO 22-2015:Masculino 54 años con ascitis maligna refractaria: uso del catéter venoso central
}

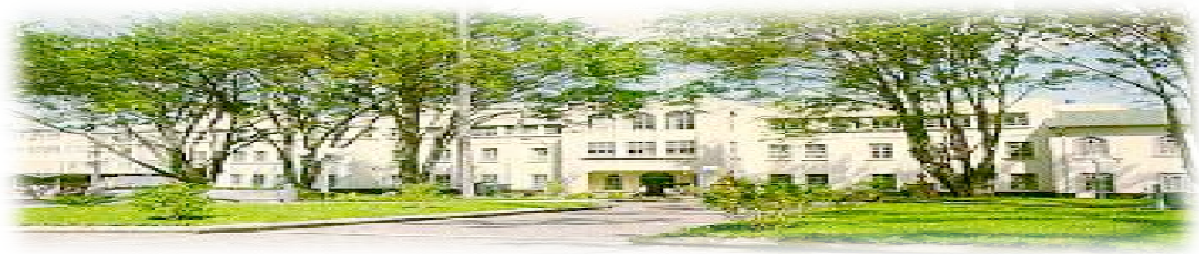

ISSN

Hospital San Juan de Dios, San José, Costa Rica. Fundado en 1845

Recibido:

$4 / 05 / 2015$

Aceptado:

$2 / 07 / 2015$

Dra. Andrea Medina Méndez ${ }^{1}$

${ }^{1}$ Medico Asistente Especialista en Medicina Paliativa de Adultos. Hospital Dr. Escalante Pradilla, Caja Costarricense del Seguro Social. andreamedinacr@hotmail.com

\section{RESUMEN}

Paciente masculino de 54 años vecino de Pérez Zeledón con antecedente de etilismo y tabaquismo y portador de una Hepatitis B y un cistoadenoma de la vía biliar. Asocio una ascitis maligna que requería una paracentesis cada 3-4 días por lo que se le coloco un catéter venoso central fenestrado para el drenaje domiciliar de su ascitis con lo que logro mejorar su calidad de vida.

\section{PALABRAS CLAVE}

Ascitis maligna, paliativo, catéter

\section{ABREVIATURAS}

AM: Ascitis Maligna

HEP: Hospital Fsco Escalante Pradilla

\begin{abstract}
Male patient,54years of Pérez Zeledón neighbor with a history of alcohol abuse, smoking and carrying a Hepatitis B and biliary cyst adenoma. Associate malignant ascites requiring paracentesis every 3-4days so that was placed fenestrated central venous catheter for house hold drain ascites there by achieving better quality of life.
\end{abstract}

\section{KEY WORDS}

Malignant ascites, palliative, catheter

\section{CASO CLÍNICO}

Paciente masculino de 54 años vecino de San Isidro de Pérez Zeledón con antecedente de etilismo y tabaquismo. Portador de Hepatitis B (desde 1993) y osteoporosis, a quién en Febrero del 2014 se le diagnosticó un cistoadenoma de la 
vía biliar. No fue candidato a cirugía y no fue valorado por oncología médica.

Llego a consulta externa de Medicina Paliativa (MP) del Hospital Dr. Escalante Pradilla (HEP) en Julio del 2014 referido de Atención Primaria para inicio de manejo paliativo. Se le inicio furosemida 40mgbid VO, espironolactona 50mg HS VO y se incluyó en el programa de Visita Domiciliar. Un mes después de la primera VD usando furosemida a $40 \mathrm{mg}$ bid $\mathrm{VO}$ y espironolactona $100 \mathrm{mg}$ HS VO se documentó que el paciente estaba asistiendo cada 3-4 días al servicio de Emergencias del HEP para que se le realizarán paracentesis evacuadoras con un Karnosky de $80 \%$. El paciente se quejaba de insomnio, dolor abdominal, náuseas, vómitos asociado a la distención abdominal que empeoraban en relación al aumento de su ascitis. En vista de que las medidas de cambio en el estilo de vida y el uso de diuréticos no lograban brindarle un alivio; se le propone la colocación de un catéter peritoneal para el manejo de dicha ascitis maligna refractaria a tensión. Tenía un gradiente albumina séri$\mathrm{ca} /$ peritoneal (SAAG): $1.9 \mathrm{~g} / \mathrm{dl}$ (alto).

El 25 de Agosto del 2014, previa firma de consentimiento informado. Se colocó un catéter venoso central (que había sido fenestrado) con técnica aséptica a nivel abdominal en punto de inserción de las paracentesis evacuadoras que se le habían realizado. El catéter se fijó de forma usual y se le adapto una conexión de suero que a su vez se adaptó a una bolsa colectora de orina. Se entrenó a los familiares a abrir y cerrar la conexión para que le drenaran el líquido ascítico dos veces al día con un máximo de 1500 cc por día.

Posterior a la colocación del catéter, paciente curso sin insomnio, sin dolor, sin estreñimiento, sin vómitos. Presento aisladas náuseas, leve anorexia y un leve hiperemia, sin secreciones sin aumento local de la temperatura y menor a 0.5 $\mathrm{cm}$ que se trató con antibiótico tópico tid.

Se suspende el catéter a los 15 días de colocados por disminución importante de la cantidad de líquido que drenaba (500cc c/24hrs) y se aumentó la dosis de los diuréticos (espironolactona $100 \mathrm{mg}$ c/8hrs y furosemida $40 \mathrm{mg}$ bid VO). Sin embargo presento una hiponatremia $(121 \mathrm{mmol} / \mathrm{L})$ secundaria a diuréticos por lo se tuvo que disminuir la dosis y la ascitis se exacerbó. Así que en conjunto se decidió colocar un nuevo catéter, furosemida $40 \mathrm{mg}$ bid VO y espironolactoana $100 \mathrm{c} / \mathrm{d}$ VO. Esté últimocatéter funcionó por 20 días y se suspendió a solicitud de paciente por que presento fuga del líquido ascítico en el punto de inserción del mismo.

Durante el tiempo que tuvo el catéter colocado; el paciente fue capaz de ir a Esparza y Ciudad Neilly a visitar a familiares sin presentar complicaciones y logrando así satisfacer sus necesidades espirituales.

\section{DISCUSIÓN Y ANÁLISIS DEL CASO}

Se habla de Ascitis Maligna (AM), cuando el acumulo de líquido ascítico en la cavidad peritoneal, está asociado a un padecimiento oncológi$\mathrm{co}^{(1,2)}$ y representa apenas el $10 \%$ de todas las $\operatorname{ascitis}^{(3-9)}$

A partir del diagnóstico de AM; el paciente tiene una esperanza de vida es de \pm 20 semanas $^{(9)}$; aunque para otros autores puede ser variable el tiempo (2-6 meses según Saiz. No existe una terapia anti-tumoral efectiva ${ }^{(9)}$.La AM se puede presentar como primer síntoma de una enfermedad oncológica en el $52-54 \%^{(3,9)}$ de los casos y de esos un $20 \%$ de todos los caso tienen un tumor primario desconocido ${ }^{(5,7,10,11) \text {. }}$

\section{Fisiopatología de la ascitis maligna}

Es compleja y multifactorial. Donde intervienen tanto la neovascularización con un aumento de la permeabilidad capilar, obstrucción en el drenaje y producción forzada de líquido peritoneal ${ }^{(12)}$. Sin olvidar el estímulo de factores como las glicoproteínas, el Factor de Crecimiento Vascular Endotelial (VEGF)

Muchos pacientes conascitis malignatieneun alto contenido deproteínas, esto va aalterar la permeabilidad vascular, estohasido implicado tambiénen la patogénesisde la ascitis ${ }^{(5)}$.

\section{Cuadro clínico}

La presentación clínica es muy variada, pero en general puede encontrarse: disnea, náuseas, anorexia, fatiga, reflujo gastroesofágico, distención abdominal, dolor abdominal, limitaciones a la movilidad, distres respiratorio, anasarca, saciedad temprana, aumento de peso, cambios en los 
hábitos intestinales y edema escrotal, en pene y periférico.

En el examen físico se puede encontrar un abombamiento de los flancos (cuando hay entre 500 y $1000 \mathrm{~mL}$ de líquido), matidez en los flancos, el signo de la ola, el signo del charco (cuando hay entre 300 y $400 \mathrm{~mL}$ de líquido), hernia abdominales, matidez a la percusión de la base del hemitoráx derecho (derrame pleural) y edemas en miembros inferiores, genitales y abdomen bajo.

La AM se puede clasificar según:

1- Por el SAAG en $<1.1$ (bajo) ó $>1.1$ (alto)

2- Por su severidad: leve, moderada, severa

3- Por la presencia de complicaciones: no complicada o complicada

4- Según la refractariedad: resistente a diuréticos o intratable a diuréticos

\section{Abordaje terapéutico}

La selección de una modalidad de tratamiento apropiado, sigue siendo un cuidadoso proceso, que debería tener en cuenta:

-El potencial riesgos

-Beneficios

-La esperanza de vida de la paciente según su pronóstico

En relación al manejo médico de la AM lo primero que se debe de hacer es clasificar la AM según SAAG y en base a esta clasificación iniciar el manejo (algoritmo 1)
Tabla 1. Algoritmo de la Ascitis Maligna

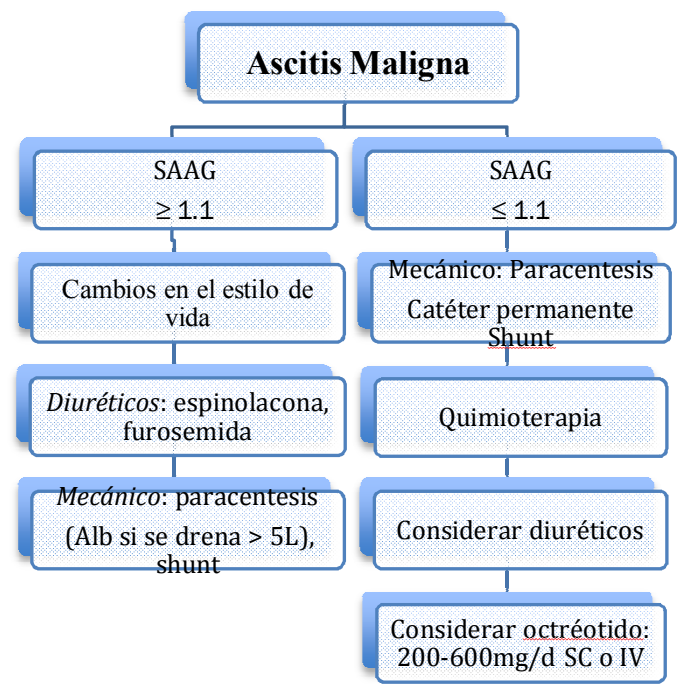

Fuente: Tarascon. Palliative Medicine Pocketbook. 2012. Pag 52 (20)

\section{Diuréticos}

$1^{\circ}$ línea de tratamiento para algunos autores. Se considera efectiva en el $40 \%$ pacientes con ascitis maligna ${ }^{(6)}$.Se logra reabsorber con los diuréticos un máximo de $800 \mathrm{~mL}$ por día con una pérdida de $1 \mathrm{~kg} /$ día. Su eficacia es mayor cuando la ascitis es $2^{\circ}$ a metástasis hepáticas con hipertensión portal e hipoalbuminemia ${ }^{(12)}$.

\section{Paracentesis}

Es la terapia más común y efectiva. Logra un alivio temporal de los síntomas en el $90 \%{ }^{(3)}$.

La severidad de los síntomas se correlaciona con la presión intraperitoneal previa al procedimiento pero no con el volumen de líquido de ascitis ${ }^{(13)}$. Antes de su realización se debe valorar el riesgo del paciente para el desarrollo de complicaciones. Es difícil predecir qué pacientes pueden presentar estas complicaciones ${ }^{(14)}$.

El alivio de los síntomas con la parecentesis suele un promedio de 10 días ${ }^{(13)}$. En los pacientes que precisan paracentesis de repetición ( $>1 \mathrm{cada}$ 7-15 días) debería plantearse el uso de catéteres permanentes $^{(14) \text {. }}$ 


\section{Catéteres:}

Para pacientes a los que hay que realizarle paracentesis frecuentes ( $<7$ días) son los que ven más beneficiados. El drenaje puede ser permanente o intermitente para así evitar la tensión.

Beneficios: autodrenaje, elimina la necesidad de hospitalización y menor disconfort.

Complicaciones: Peritonitis, celulitis, fuga en el sitio de punción infección del catéter, oclusión del catéter, fuga del catéter e hipotensión severa.

Contraindicaciones: síndrome adherencial y carcinomatosis y líquido loculado

Existen muchas opciones de catéteres: "pigtail," pleurX, Tenckhoff, port-a-cath, de diálisis (tunelizados). La mayoría de los autores prefieren los catéteres tunelizados porque asocian un menor riesgo de infección y tienen mayor estabilidad que los "pig-tail" estándares.La Food and DrugAdministration (FDA) aprobó enel 2005 el uso de los catéteres Pleur X para el manejo de la $\mathrm{AM}^{(1)}$.

La infección del catéter está más asociada a la duración por lo que debe de usarse en pacientes con expectativa de vida de semanas y no meses. Se entrena al paciente para que se drena a diario por las primeras 2 semanas y luego $\mathrm{PRN}^{(7) \text {. }}$

\section{CONCLUSIONES}

La aparición de la AM le va a dar una gran gama de sintomatología al paciente que lo hará consultar múltiples veces en los servicios de emergencias; lo cual le limitara su calidad de vida y donde el uso de diuréticos y los cambios en el estilo de vida tienen una aplicación limitada. Por lo que se hace necesario buscar alternativas terapéuticas como el uso de catéteres peritoneales para que el paciente recupere su calidad de vida.

\section{BIBLIOGRAFÍA}

1. Prommer E Ammouri L. Palliative treatment of malignant ascites: profile of catumaxomab. Biol Targets Ther. mayo de 2010;103.
2. Guillén et all. Manejo Paliativo de la Ascitis en Pacientes Oncológicos. Cancerologìa. 2008;3:149-57.

3. Saif M Siddiqui IP Sohail M. Management of ascites due to gastrointestinal malignancy. Ann Saudi Med. 2009;29(5):369.

4. Tewari K Eskander RN. Emerging treatment options for management of malignant ascites in patients with ovarian cancer. Int $\mathrm{J}$ Womens Health. agosto de 2012;395.

5. Becker G Galandi D Blum HE. Malignant ascites: Systematic review and guideline for treatment. Eur J Cancer. marzo de 2006;42(5):589-97.

6. Chung M Kozuch P. Treatment of Malignant Ascites. Curr Treat Options Oncol. junio de 2008;9(2-3):215-33.

7. Rosenberg SM. Palliation of Malignant Ascites. GastroenterolClin North Am. marzo de 2006;35(1):189-99.

8. Saiz-Mendiguren R, Gómez-Ayechu M, Noguera JJ et al. Drenaje permanente tunelizado de la ascitis maligna: experiencia inicial con el catéter Pleur $X{ }^{\circledR}$. Radiología. noviembre de 2010;52(6):541-5.

9. Sangisetty SL. Malignant ascites: A review of prognostic factors, pathophysiology and therapeutic measures. World J GastrointestSurg. 2012;4(4):87.

10. BerchídDébdi M Martínez Chaves V Ceacero Rodríguez C Medina Muñoz J. Paracentesis evacuadora domiciliaria en cuidados paliativos realizada por profesionales de atención primaria. Presentación de un caso. SEMERGEN - Med Fam. abril de 2013;39(3):161-4.

11. Sales Moreno P Calsina-Berna A López Postigo M et I. Estudio descriptivo sobre el uso de catéteres peritoneales en el manejo de la ascitis maligna. Med Paliativa. enero de 2012;19(1):38-44.

12. Cavazzoni E Bugiantella W Graziosi L Franceschini MS Donini A. Malignant ascites: pathophysiology and treatment. Int J ClinOncol. febrero de 2013;18(1):1-9.

13. Mullard AP Bishop JM Jibani M. Intractable Malignant Ascites: An Alternative Management Option. J PalliatMed. febrero de 2011;14(2):2513.

14. Salinas A Benitez M. Diagnóstico y tratamiento de la ascitis en pacientes con càncer en fase terminal. Med Paliativa. 2006;13(2):94-9. 
CONFLICTO DE INTERÉS Y/O AGRADECIMIENTOS

Los autores declaran que no existió ningún conflicto de interés en el presente reporte. 